\title{
TOWARDS A WZ EVOLUTION OF THE MEHTA INTEGRAL*
}

\section{DORON ZEILBERGER ${ }^{\dagger}$}

Abstract. The celebrated Mehta integral is shown to be equivalent to a simple algebraicdifferential identity, which is completely routine for any fixed number of variables.

Key words. WZ form, computer-assisted proof of identities

AMS subject classifications. 33A30, 33A99

Askey [As] proposed the problem of proving the Mehta (see [M]) integral identity

(Mehta)

$\frac{1}{(2 \pi)^{n / 2}} \int_{-\infty}^{\infty} \cdots \int_{-\infty}^{\infty} \exp \left(-x_{1}^{2} / 2-\cdots-x_{n}^{2} / 2\right) \prod_{1 \leq i<j \leq n}\left(x_{i}-x_{j}\right)^{2 c} d x_{1} \cdots d x_{n}=\prod_{j=1}^{n} \frac{(c j) !}{j !}$

without using Selberg's integral (see [M]). This problem was solved by Anderson [An]. Here we use the method of [WZ1] and [WZ2] to initiate another Selberg-free proof that we believe is of independent interest. We show that (Mehta) for any given $n$ is equivalent to the following elegant identity $(d:=n(n-1) / 2)$ :

(Mehta')

$$
\left\{\sum_{i=1}^{n} \frac{\partial^{2}}{\partial x_{i}^{2}}+2 c \sum_{1 \leq i<j \leq n} \frac{1}{x_{i}-x_{j}}\left(\frac{\partial}{\partial x_{i}}-\frac{\partial}{\partial x_{j}}\right)\right\}^{d} \prod_{1 \leq i<j \leq n}\left(x_{i}-x_{j}\right)^{2}=2^{d} d ! n !
$$

$\left(\mathrm{Mehta}^{\prime}\right)$ is purely routine for any specific $n$, but at the time of writing we are unable to prove it directly for general $n$. Of course, we do have $a$ proof, since we are going to show that (Mehta') and (Mehta) are equivalent, but what we are after is a direct proof. The author is offering a prize of 25 US dollars for such a proof.

The present method also obviously extends to the Macdonald-Mehta integral [M], which was proved by Beckner and Regev $[\mathrm{BR}]$ for the classical root systems (see $[\mathrm{M}]$ ), by Garvan [G] for the exceptional root system $F_{4}$, and by Opdam [O] for $E_{6}, E_{7}$, and $E_{8}$. It follows that the present approach should also yield new proofs for all the exceptional root systems, at least in principle, but most likely in practice also. More important, it seems to have a high chance of producing a uniform, intrinsic, classification-independent proof. We leave to the reader, as an instructive exercise, the task of finding the root-system analog of $\left(\mathrm{Mehta}^{\prime}\right)$ that is equivalent to the nowproved Mehta-Macdonald conjecture, and we offer an additional 25 dollars for an intrinsic proof.

Our proposed proof of (Mehta) will be a derivation rather than a verification, and will follow the method of [WZ2]. Let us call the left of (Mehta) $L(c)$, and the integrand $F\left(c ; x_{1}, \ldots, x_{n}\right)$. We know from the general theory of [WZ2] that for some $r$

* Received by the editors April 2, 1992; accepted for publication (in revised form) May 11, 1993.

$\dagger$ Department of Mathematics, Temple University, Philadelphia, Pennsylvania 19122. This work was supported in part by the National Science Foundation. 
and some rational functions $P_{1}, \ldots, P_{n}$, in $\left(c, x_{1}, \ldots, x_{n}\right)$, and some rational functions in $c, a_{0}(c), \ldots, a_{r}(c)$,

$$
\sum_{s=0}^{r} a_{s}(c) F\left(c+s ; x_{1}, \ldots, x_{n}\right)=\sum_{i=1}^{n} \frac{\partial}{\partial x_{i}}\left(P_{i} F\right) .
$$

Let us be optimistic and try out $r=1$. Without loss of generality, set $a_{1}:=1$. Substituting $F$ in (WZ), performing all differentiations, and dividing throughout by $F$ leads to the following equation for $P_{i}$ and $a_{0}$ :

$$
a_{0}+\prod_{1 \leq i<j \leq n}\left(x_{i}-x_{j}\right)^{2}=\sum_{i=1}^{n} \frac{\partial P_{i}}{\partial x_{i}}+2 c \sum_{i=1}^{n}\left(\sum_{j \neq i} \frac{1}{x_{i}-x_{j}}\right) P_{i}-\sum_{i=1}^{n} x_{i} P_{i} .
$$

To be even more optimistic, assume that $P_{i}$ are polynomials, rather than mere rational functions, in their dependence on $\left(x_{1}, \ldots, x_{n}\right)$, and are furthermore the components of the gradient of another polynomial $P$, i.e., $P_{i}=\partial P / \partial x_{i}, i=1, \ldots, n$, for some polynomial $P$. Equation (1) then becomes

$$
a_{0}+\prod_{1 \leq i<j \leq n}\left(x_{i}-x_{j}\right)^{2}=\left\{\sum_{i=1}^{n} \frac{\partial^{2}}{\partial x_{i}^{2}}+2 c \sum_{i=1}^{n}\left(\sum_{j \neq i} \frac{1}{x_{i}-x_{j}}\right) \frac{\partial}{\partial x_{i}}\right\} P-\sum_{i=1}^{n}\left(x_{i} \frac{\partial}{\partial x_{i}}\right) P .
$$

If we can find such a polynomial $P$, and compute the corresponding $a_{0}$, then it would follow from (WZ), upon integrating with respect to $x_{1}, \ldots, x_{n}$, that $L(c)$ satisfies the recurrence $L(c+1)=-a_{0}(c) L(c)$, which combined with $L(0)=1$ would enable one to find $L(c)$. Note that the mere existence of $a_{0}$, which we will shortly prove, is given by the left side of $\left(\mathrm{Mehta}^{\prime}\right)$ and implies that $L(c)$ is of closed form, which from a theoretical point of view is almost as good as knowing what it is exactly.

Let us write $P$ as a sum of its homogeneous parts

$$
P=\sum_{j=2}^{2 d} P^{(j)}, \quad P^{(j)} \quad \text { homog. of deg. } j
$$

where, as above, $d$ equals $n(n-1) / 2$. Denote the operator inside the braces of (2) or (Mehta') by Z. Using Euler's formula, we get

(3)

$a_{0}+\prod_{1 \leq i<j \leq n}\left(x_{i}-x_{j}\right)^{2}=\sum_{j=2}^{2 d} \mathrm{Z} P^{(j)}-\sum_{j=2}^{2 d} j P^{(j)}=(-2 d) P^{(2 d)}+\sum_{j=2}^{2 d}\left(\mathrm{Z} P^{(j)}-(j-2) P^{(j-2)}\right)$.

By equating corresponding homogeneous parts, we get

$$
P^{(2 d)}=-(2 d)^{-1} \prod_{1 \leq i<j \leq n}\left(x_{i}-x_{j}\right)^{2}, \quad P^{(j-2)}=\frac{1}{j-2} \mathbf{Z} P^{(j)}, \quad j=2 d, 2 d-2, \ldots, 4 .
$$


It is easy to see that $\mathbf{Z}$ maps homogeneous symmetric polynomials to homogeneous symmetric polynomials, and that it reduces the degree by 2 . Iterating (4) and comparing the constant part of (3) finally yields that both $P$ and $a_{0}$ indeed exist, and that

$$
a_{0}=-\left(2^{d} d !\right)^{-1} \mathbf{Z}^{d}\left[\prod_{1 \leq i<j \leq n}\left(x_{i}-x_{j}\right)^{2}\right] .
$$

Hence Mehta's integral is indeed expressible in closed form, and proving that its value coincides with the value implied by (Mehta) amounts to proving (Mehta').

The referee empirically noticed the following generalization. For $a$, any positive integer, we have

$$
\begin{gathered}
\left(\text { Mehta }^{\prime \prime}\right) \quad\left\{\sum_{i=1}^{n} \frac{\partial^{2}}{\partial x_{i}^{2}}+2 c \sum_{1 \leq i<j \leq n} \frac{1}{x_{i}-x_{j}}\left(\frac{\partial}{\partial x_{i}}-\frac{\partial}{\partial x_{j}}\right)\right\}^{a d} \prod_{1 \leq i<j \leq n}\left(x_{i}-x_{j}\right)^{2 a} \\
=2^{a d}(d a) ! n ! a \prod_{2 \leq r \leq n} \prod_{1 \leq s \leq r a, s \neq 0 \bmod r}(r c+s) .
\end{gathered}
$$

It turns out that (Mehta") follows from (Mehta) exactly the same way as (Mehta'); just replace $c$ by $c a$, in (Mehta), and repeat the argument! In particular, it follows that (Mehta') implies (Mehta"), albeit indirectly, via (Mehta).

Acknowledgment. Many thanks are due to Herb Wilf for countless discussions and inspiration.

\section{REFERENCES}

[An] G. Anderson, Letter to R. Askey.

[As] R. A. AskeY, Lost and found mathematics, joint MAA-AAAS invited talk, Columbus, OH, August 1990.

[BR] W. BECKNER AND A. REGEV, manuscript, 1980.

[G] F. G. Garvan, Some Macdonald-Mehta integrals by brute force, in q-Series and Partitions, D. Stanton, ed., IMA volumes 18, Springer-Verlag, New York, pp. 77-98.

[M] I. G. MACDONALD, Some conjectures for root systems, SIAM J. Math. Anal., 13 (1982), pp. 988-1007.

[O] E. OPDAM, Some applications of hypergeometric shift operators, Invent. Math., 98 (1989), pp. 1-18.

[WZ1] H. S. WILF AND D. ZEILBERGER, Rational function certification of hypergeometric multiintegral/sum/“ $q$ " identities, Bull. Amer. Math. Soc., 24 (1992), pp. 143-148.

[WZ2] - , An algorithmic proof theory for hypergeometric (ordinary and " $q$ ") multisum/integral identities, Invent. Math., 108 (1992), pp. 575-633. 